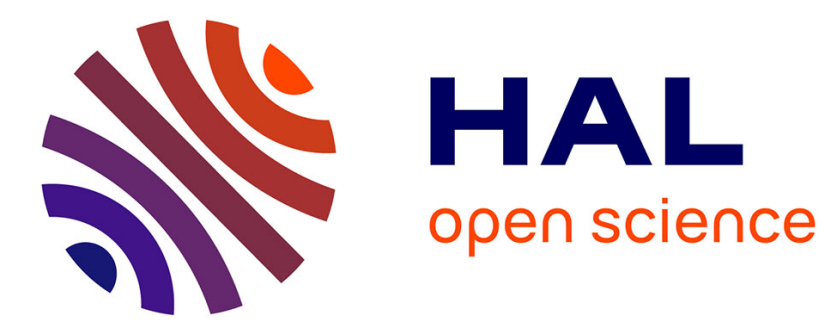

\title{
Harmonie des observations in-situ STM et électrochimiques sur électrodes monocristallines d'or
}

\author{
A. Hamelin
}

\section{To cite this version:}

A. Hamelin. Harmonie des observations in-situ STM et électrochimiques sur électrodes monocristallines d'or. Journal de Physique IV Proceedings, 1994, 04 (C1), pp.C1-315-C1-322. 10.1051/jp4:1994124 . jpa-00252466

\section{HAL Id: jpa-00252466 https://hal.science/jpa-00252466}

Submitted on 1 Jan 1994

HAL is a multi-disciplinary open access archive for the deposit and dissemination of scientific research documents, whether they are published or not. The documents may come from teaching and research institutions in France or abroad, or from public or private research centers.
L'archive ouverte pluridisciplinaire HAL, est destinée au dépôt et à la diffusion de documents scientifiques de niveau recherche, publiés ou non, émanant des établissements d'enseignement et de recherche français ou étrangers, des laboratoires publics ou privés. 


\title{
Harmonie des observations in-situ STM et électrochimiques sur électrodes monocristallines d'or
}

\author{
A. HAMELIN \\ Laboratoire d'Electrochimie des Interfaces, CNRS, 92195 Meudon, France
}

RESUME

A partir de l'observation d'une hystérèse ou d'une dispersion avec la fréquence du signal alternatif, sur les courbes capacité différentiellepotentiel, il a été suggéré que les surfaces des plans (100), (111) et (110) de l'or changent d'arrangement atomique à I'interface électrochimique dans la région dite de couche double. En collaboration avec X. Gao et M. J. Weaver des images de ces plans de l'or ont été obtenues in situ par Microscopie à Effet Tunnel. La nature et la cinétique de ces changements de structures atomiques superficielles avec la charge à l'électrode sont décrites.

\section{ABSTRACT}

From observations of hysteresis or alternative signal frequency dispersion on differential capacity-potential curves, it was suggested that (100), (111) and (110) gold faces change their atomic superficial arrangements at the electrochemical interface in the double layer region of potential. In situ STM images of these gold faces obtained with X.Gao and M. J. Weaver allowed to describe the nature and the kinetics of these superficial atomic structural changes.

Pour l'or par la voltammétrie cyclique, VC, on peut discerner trois régions de potentiel (fig.1), une région dite de couche double, A, où les interactions sont purement électrostatiques, une région où ill $\mathrm{y}$ a formation et réduction d'une monocouche de composés oxygénés, $B$, et une région $C$ où on observe le début de la réduction du solvant, l'eau. Si on superpose au potentiel imposé, qui varie linéairement avec le temps, un signal alternatif de faible amplitude on peut observer dans la région A les variations de la capacité différentielle, $C$, en fonction du potentiel imposé, $E$. Dans ce domaine de potentiel $A$, le modèle de couche double s'applique. Lorsqu'il n'y a pas d'adsorption, on observe en solutions diluées la contribution de la partie diffuse de la couche double, $C_{d}$, qui. s'accentue avec la dilution de la solution (le potentiel au minimum correspond au potentiel de charge nulle, pzc), et en solutions concentrées on observe principalement la contribution de la partie interne de la couche double. 
Dès 1974 il a été observé que ces courbes C(E) enregistrées en régime potentiodynamique ( 3 à $8 \mathrm{mV} / \mathrm{s}$ ) dépendent, pour les faces (100) et (111) de l'or, des limites de l'exploration en potentiel. Pour le plan (210) on n'observe pas ce phénomène. Pour (110) il y a peu d'influence des bornes dé potentiel, mais un dispersion des valeurs de $\mathbf{C}$, dans un certain domaine de potentiel, en fonction de la fréquence du signal alternatif.

Nos résultats obtenus avant 1984 sont résumés réf. 1 et 2 . A titre d'exemple voici quelques courbes obtenues par la suite. Pour (100) en acide perchlorique dilué il y a clairement un effet de la borne négative sur les valeurs de capacité et le pzc ( $f i g .2$ a). Des résultats similaires en milieux neutres, obtenus des années avant, avaient déjà suggéré un changement de structure atomique superficielle lorsque des densités de charge négatives existent à la surface de ce plan, changements qui redisparaissent à charges nettement positives.

Pour (111) en acide perchlorique il y a une influence de la borne positive de potentiel clairement visible en milieu dilué ( $f i g .2 \mathrm{~b}$ ).

Lorsqu'il y a adsorption spécifique, une hystérèse entre les balayages positif et négatif des courbes $C(E)$ est observée pour (100) et (111) (fig.3), pour (110) une dispersion avec la fréquence existe (fig.4).Pour (210) aucun de ces phénomènes n'a été observé.

L'ensemble de nos résultats électrochimiques obtenus pour les faces d'or, lors d'un balayage permanent du domaine de potentiel, suggère, pour les plans d'indices simples de l'or, une structure superficielle (1X1) (c'est à dire semblable à celle qui existe dans un plan parallèle à la surface au sein du métal) à charges nettement positives sur le métal, et une structure différente dite "reconstruite" aux charges négatives. Ceci n'est pas observé pour l'or (210) ou des monocristaux d'argent étudiés dans des conditions similaires. En 1990 j'aj tenté de résumer nos résultats [3].

Dès 1991 à Purdue University, West Lafayette, IN (USA) avec X. Gao et M.J.Weaver il a été possible d'obtenir, in-situ en 0.1 M $\mathrm{HClO}_{4}$ par Microscopie à Effet Tunnel (STM) des images qui permettent l'identification de chaque atome d'or à la surface de l'électrode, pour un potentiel imposé potentiostatiquement. 


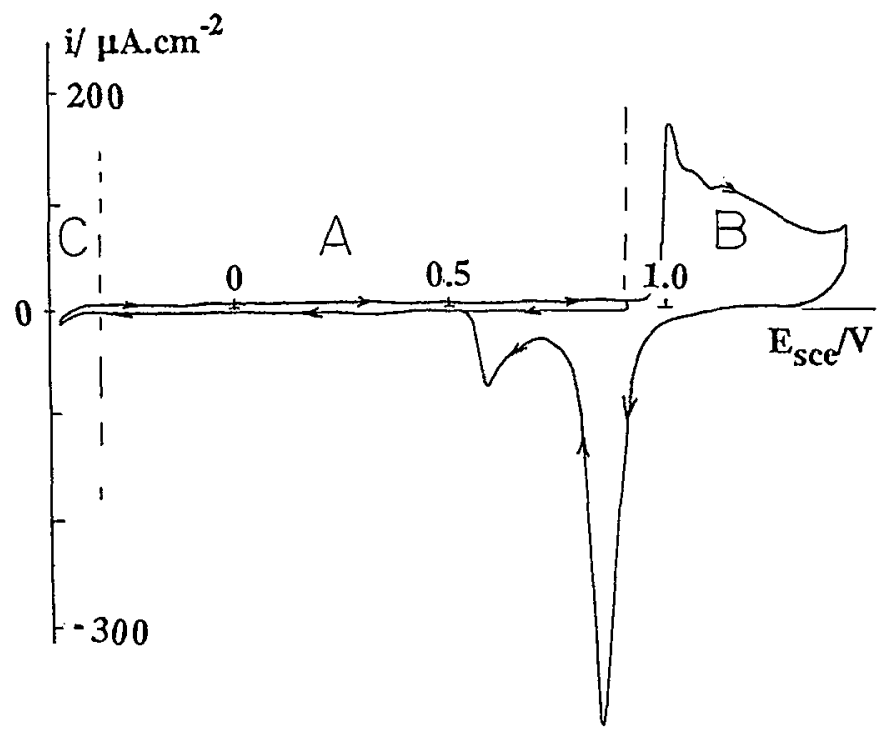

Fig. 1 - VC pour l'or (110) en $0.1 \mathrm{M} \mathrm{HClO}_{4}$ à $23^{\circ} \mathrm{C} ; 80 \mathrm{mV} / \mathrm{s} ;$ I'électrode de référence était une électrode réversible à hydrogène, RHE, mais ici les potentiels sont donnés par rapport à une électrode à calomel saturée, SCE.
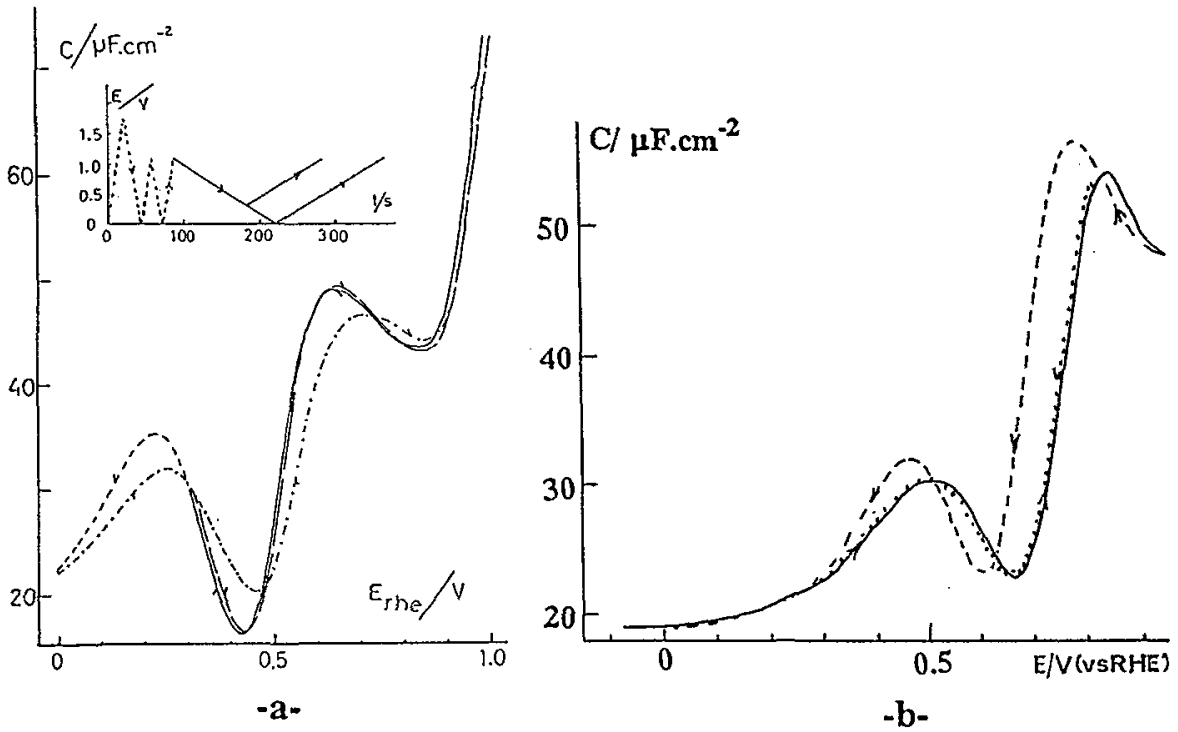

Fig. 2 - Courbes C(E):

-a- pour (100) en $0.01 \mathrm{M} \mathrm{HClO}_{4}\left(20 \mathrm{~Hz}, 5 \mathrm{mV} / \mathrm{s}, 25^{\circ} \mathrm{C}\right)$ enregistrées au cours d'un balayage permanent comprenant l'étape B. Encart: programme potentiel-temps.

-b-pour $(111)$ en $0.006 \mathrm{M} \mathrm{HClO}_{4}\left(20 \mathrm{~Hz}, 8 \mathrm{mV} / \mathrm{s}, 35.8^{\circ} \mathrm{C}\right)$. L'électrode n'a pas été soumise à l'étape $B$ qui crée des défauts sur cette face. Dans ce cas 0 SCE est à $0.376 \mathrm{~V}$ par rapport à RHE. 


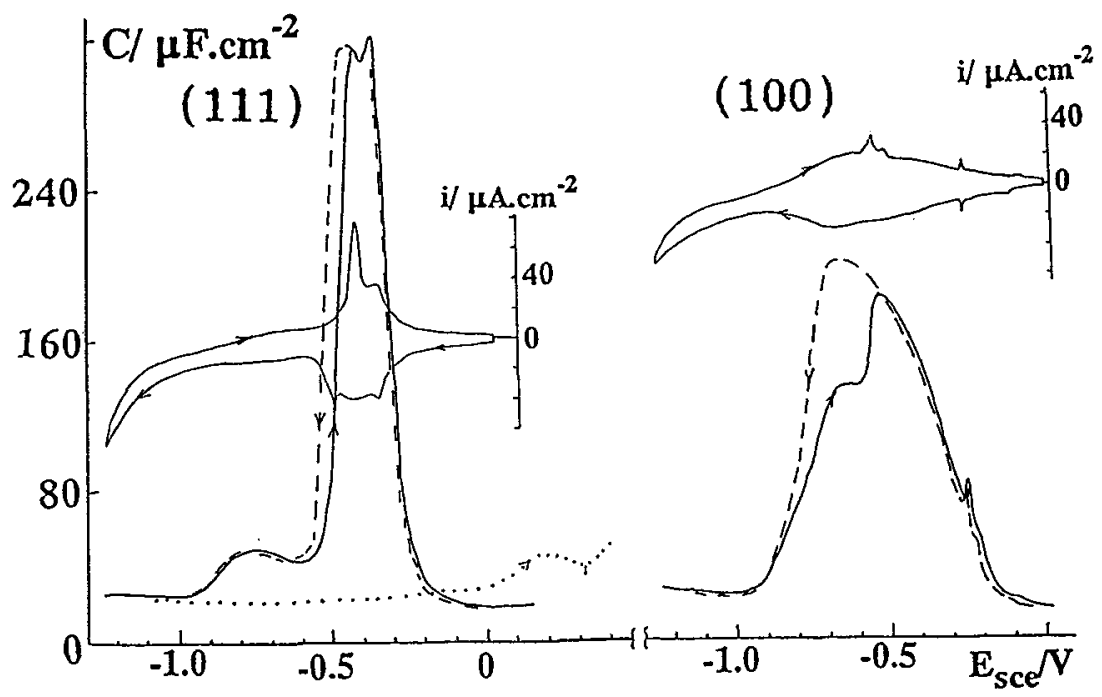

Fig. 3 - Courbes $C(E)$ pour (111) et (100) en $0.1 \mathrm{M} \mathrm{NaF}+0.5 \mathrm{mM} \mathrm{NaI} \mathrm{(2O} \mathrm{Hz,8}$ $\mathrm{mV} / \mathrm{s}$ ) et VC correspondants. (...) $\mathrm{C}(\mathrm{E})$ en $0.02 \mathrm{M}$ NaFpour (111) donnée pour comparaison.
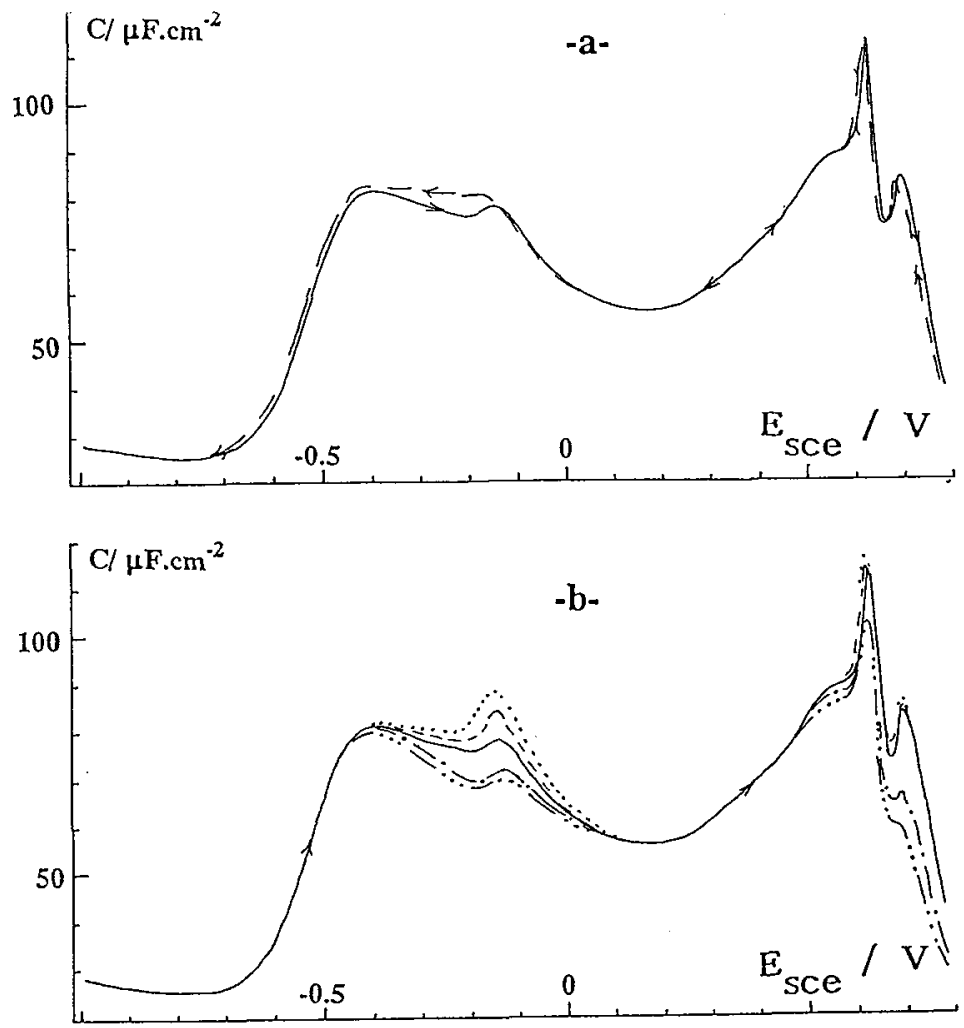

Fig. 4 - C(E) pour (110) en 0.1 M NaCl: a - balayages positif et négatif (20 $\mathrm{Hz}, 5 \mathrm{mV} / \mathrm{s}$ ); b - balayages positifs pour $8,12,20$, 80 et $180 \mathrm{~Hz}$. 
Ces observations ont été faites sur des faces monocristallines préparées et vérifiées par VC en $\mathrm{HClO}_{4} \mathrm{O} .01 \mathrm{M}$ au LEI, traitées à la flamme d'un chalumeau gaz-oxygène et refroidies dans de l'eau ultra-pure juste avant d'être examinées par STM (c'est à dire sans contact avec l'air ambiant).

Pour les trois plans d'indices simples de l'or à charges nettement négatives la structure atomique superficielle n'est pas celle qui existe au sein du métal dans un plan parallèle à la surface mais un structure dite "reconstruite", tandis que à charge nettement positive la structure superficielle atomique est (1X1). En revenant à charges négatives on reforme les structures reconstruites (fig.5), plus ou moins rapidement suivant l'orientation cristallographique: lentement (de l'ordre d'une dizaine de minutes) pour (100) et (111), beaucoup plus rapidement pour (110) (de l'ordre de la seconde). La description complète de ces surfaces "reconstruites" ne peut être donnée ici, le lecteur se référera aux figures des réf. 4 à 7 . Disons seulement que la structure reconstruite est (5X27) pour (100), (1X22) pour (111) et pour (110) un mélange de régions (1X2) et (1X3). Ces reconstructions observées in-situ pour les plans d'indices simples de l'or sont proches de celles observées dans l'air ou dans le vide.

Par contre pour le plan (210), par STM in-situ, seulement une faible relaxation perpendiculairement à la surfaces est observée.

Ces premiers résultats ont conduit à de nouveaux résultats électrochimiques. Par exemple : si il faut environ 10 minutes pour une reconstruction complète de l'or (100), l'observation de l'évolution des courbes $\mathrm{C}(\mathrm{E})$ après avoir maintenu le potentiel $\mathrm{O}, 5,10$ minutes à la borne négative doit reflèter cette reconstruction. En effet, pour (100) en $\mathrm{HClO}_{4} 1$ $\mathrm{mM}$ on observe un évasement progressif de la contribution de la partie diffuse de la couche double qui n'est pas observé pour (210) (fig.6) [9]. Cet évasement progressif de la contribution de la partie diffuse de la couche double correspond à une surface de plus en plus hétérogène au cours de la reconstruction de (100); en effet il se forme des facettes atomiques denses et des rangées et des doubles rangées de 13 ou 14 atomes [4]. 


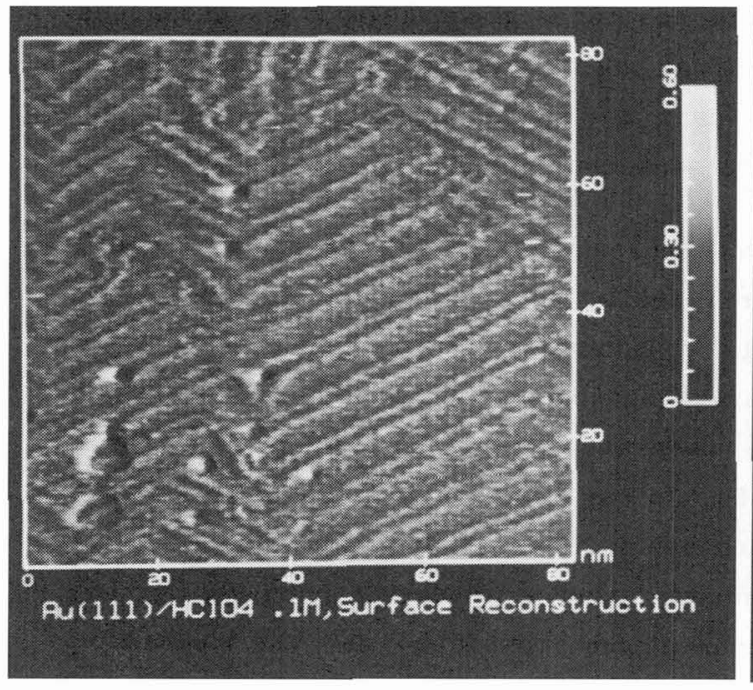

$-\mathbf{a}-$

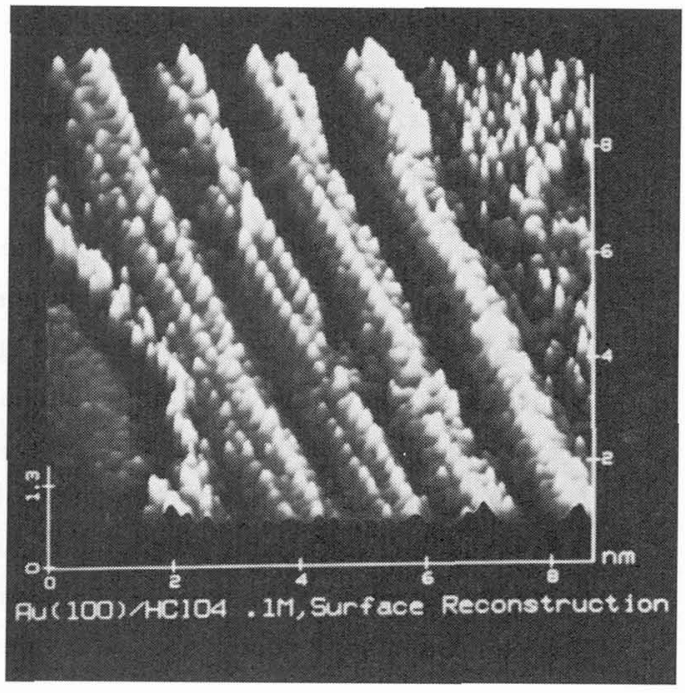

$-b-$

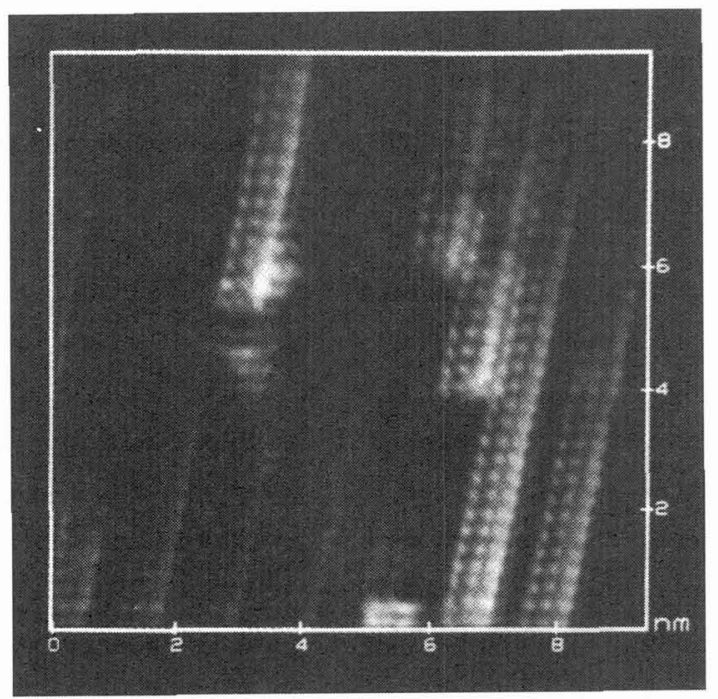

$-c-$

Fig. 5 - Images de $\mathrm{Au}(\mathrm{hkl})$ reconstruit en $0.1 \mathrm{M} \mathrm{HClO}_{4}$ :
$\mathrm{a}-(111) \grave{a}-0.3 \mathrm{~V}$ (SCE), $i_{t}=3 \mathrm{nA}, \mathrm{V}_{\mathrm{b}}=-47 \mathrm{mV}$;
$\mathrm{b}-(100) \mathrm{a}-0.4 \mathrm{~V}(\mathrm{SCE}), \mathrm{i}_{\mathrm{t}}=5 \mathrm{nA}, \mathrm{V}_{\mathrm{b}}=5 \mathrm{mV}$;
c $-(110)$ à $-0.3 \mathrm{~V}$ (SCE), on voit des domaines (1X2) et (1X3). 

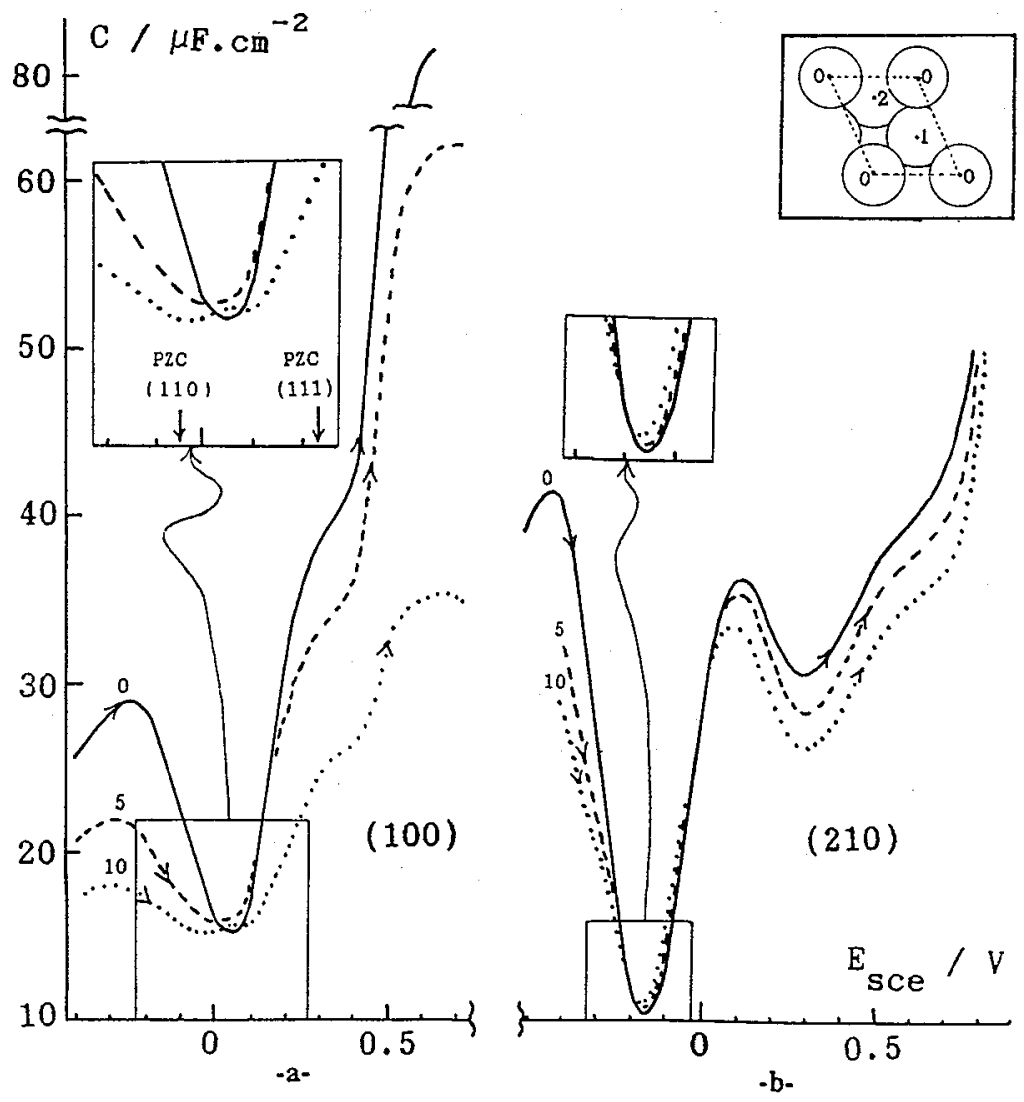

Fig.6 - C(E) pour (100)(a) et (210)(b) en $\mathrm{HClO}_{4} 1 \mathrm{mM}$ (balayages positifs, $20 \mathrm{~Hz}, 5 \mathrm{mV} / \mathrm{s}$ ) après avoir maintenu le potentiel :0, 5, 10 minutes à $-0.4 \mathrm{~V}$ (SCE). Encart: modèle du plan (210).

Par la suite des images STM ont été obtenues pour d'autres programmes potentiel-temps. Les images $\mathrm{x}, \mathrm{y}$ (fig. 5 par exemple) sont obtenues en une vingtaine de secondes, ce sont en fait des images $x$, temps obtenues à potentiels fixes. X.Gao et $M$.J.Weaver ont imposé un saut de potentiel pendant l'enregistrement d'une image, ainsi figure 2 b et $c$ de réf. 8 (pour (111) en solution $0.1 \mathrm{M} \mathrm{HClO}_{4}+0.5 \mathrm{mM} \mathrm{KI}$ ) on a une image composite, le saut de potentiel de $-0.4 \mathrm{~V}$ à $0.1 \mathrm{~V}$ par rapport à SCE ayant eu lieu au milieu de l'enregistrement de l'image; donc la moitié basse de la figure est l'image du substrat, l'or (111), la moitié haute représente l'adsorbat ayant une autre symétrie. 
Une autre tactique est de faire varier linéairement avec le temps le potentiel imposé à l'électrode d'or, au cours de l'enregistrement de l'image STM, on a alors une image $x, E$. Cette possibilité a fourni des précisions sur la façon dont le plan (100) passe de la structure dite reconstruite à la structure (1X1) par l'intermédiaire de la formation d'îlots plus ou moins grands suivant le milieu (perchlorique ou sulfurique) (figure 4 de réf.9).

Pour conclure, on constate l'harmonie des résultats obtenus in-situ par Microscopie à Effet Tunnel, par voltammétrie cyclique, par courbes capacité différentielle-potentiel, pour les plans de l'or en solutions aqueuses.

\section{REFERENCES}

[1] Hamelin A., J.Electroanal. Chem., 142 (1982) 299.

[2] Hamelin A. dans Modern Aspects of Electrochemistry, vol. 16, B. E. Conway, R. E. White et J.O'M. Bockris, eds, Plenum Press, N.Y., 1985, chap. 1.

[3] Hamel in A., J.Chim. Phys., 88 (1991) 1453.

[4] Gao X., Hamelin A., Weaver M. J., Phys. Rev. Lett., 67, n5 (1991) 618.

[5] Gao X., Hamelin A., Weaver M. J., Phys. Rev. B, 46 (1992) 7096.

[6] Gao X, Hamelin A., Weaver M. J., Phys. Rev. B, 444, n¹9 (1991) 10983.

[7] Gao X., Hamel in A., Weaver M. J., J.Chem. Phys., 95, n॰9 (1991) 6993.

[8] Gao X., Weaver M. J., J. Am. Chem. Soc, , 114 (1992) 8544.

[9] Hamelin A., Stoicoviciu L., Edens G., Gao X., Weaver M.J., J. Electroanal. Chem. (1993) accepté pour publication. 